\title{
Le travail des femmes dans le secteur agricole: Entre précarité et empowerment-Cas de trois régions en Egypte, au Maroc et en Tunisie
}

Zhour Bouzidi

Saker El Nour

Wided Moumen

Follow this and additional works at: https://knowledgecommons.popcouncil.org/departments_sbsr-pgy

Part of the Demography, Population, and Ecology Commons, Family, Life Course, and Society Commons, Gender and Sexuality Commons, International Public Health Commons, Labor Economics Commons, and the Work, Economy and Organizations Commons How does access to this work benefit you? Let us know!

\section{Recommended Citation}

Bouzidi, Zhour, Saker El Nour, and Wided Moumen. 2011. "Le travail des femmes dans le secteur agricole: Entre précarité et empowerment-Cas de trois régions en Egypte, au Maroc et en Tunisie," Gender and Work in the MENA Region Working Paper no. 22. Cairo: Population Council. 
NUMBER 22

JUNE 2011

GENDER AND WORK IN THE MENA REGION

WORKING PAPER SERIES

Poverty, Job Quality and Labor Market Dynamics

Le travail des femmes dans le secteur agricole: Entre précarité et empowerment

Cas de trois régions en Egypte, au Maroc et en Tunisie

Z. Bouzidi S. El Nour W. Moumen

(2) Population Council 
Zhour Bouzidi, LADYSS, université Paris Ouest Nanterre la défense- France, Ecole Nationale d'Agriculture de Meknès-Maroc. Email: zhour_bouzidi@yahoo.fr

Saker EINour, Université de la vallée sud- Egypte, GECKO, université Paris Ouest Nanterre la défenseFrance. Email: sakerabdo@@gmail.com

Wided Moumen, Université de Toulouse- France. E-mail : moumen_wided@yahoo.fr Ce document traite de projets appuyépar le Centre de recherches pour le développement international, organism canadien (www.crdi.ca).

\section{IDRC * CRDI}

Canadä

\section{Population Council}

Research that makes a difference

Le Population Council s'attelle aux questions cruciales de santé et de développement -quili s'agisse d'endiguer la propagation du VIH, d'améliorer la santé reproductive et d'assurer que les jeunes mènent une vie satisfaisante et productive. Grâce à des travaux de recherche en biomédecine, en sciences sociales et en santé publique dans environ 50 pays, le Population Council travaille avec ses partenaires pour offrir des solutions qui conduisent à des politiques, des programmes et des technologies plus efficaces pour améliorer la vie partout dans le monde. Fondé en 1952 et basé à New York, le Population Council est une organisation non gouvernementale à but non lucratif avec un conseil d'administration international.

Population Council

Egypt Office

59 Misr Helwan Agricultural Road, Maadi, Cairo, Egypt

Tel.: (+202) 2525-5965, (+202) 2525-5967, (+202) 2525-5968

Facsimile: (+202) 2525-5962

Website: http://www.popcouncil.org

E-mail: pcouncil@popcouncil.org

(c) 2011 The Population Council, Inc.

Toute partie de ce document peut être reproduite en quantité limitée sans la permission du Population Council pourvu quélle soit distribuée gratuitement ou à des fins non lucrative et que la source soit identifiée. Le Population Council voudraient bien recevoir une copie de tous les matériaux qui emploient le texte.

ISSN: 11882/2011 
NUMBER 22

JUNE 2011

GENDER AND WORK IN THE MENA REGION WORKING PAPER SERIES

Poverty, Job Quality and Labor Market Dynamics

Le travail des femmes dans le secteur agricole: Entre précarité et empowerment

Cas de trois régions en Egypte, au Maroc et en Tunisie

Z. Bouzidi S. El Nour W. Moumen

(2) Population Council 



\section{Table of contents}

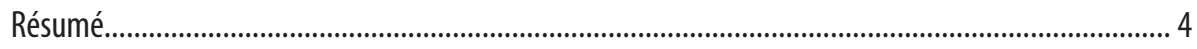

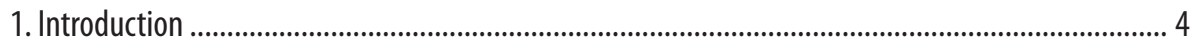

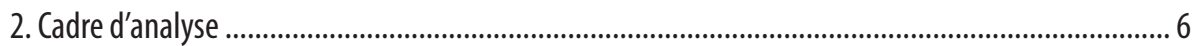

2.1 Deux dimensions pour appréhender le travail de femmes en agriculture .............................. 6

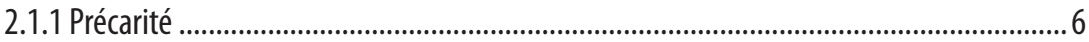

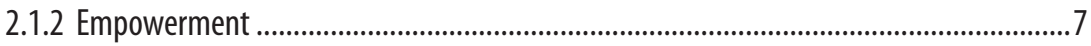

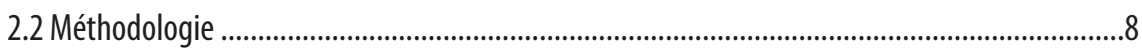

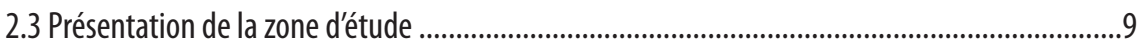

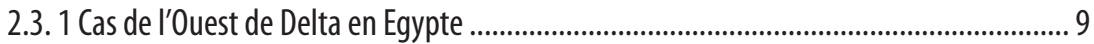

2.3.2 Cas de la zone côtière du Gharb au Maroc .....................................................................9

2.3.3 Cas de la délégation de Nadhour enTunisie ..............................................................10

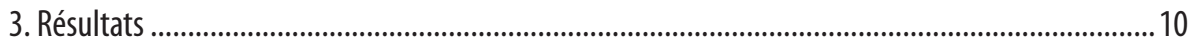

3.1 Contexte et conditions du travail: Précarité générale dans les trois contextes ........................ 10

3.1.1 La région de l'ouest de Delta en Egypte ......................................................................10

3.1.2 La zone côtière du Gharb au Maroc ............................................................................... 11

3.1.3 La région de Nadhour en Tunisie ................................................................................. 13

3.2 Quelques éléments de l'empowerment des ouvrières ..........................................................14

3.2.1 La région de l'ouest de Delta en Egypte ...................................................................14

3.2.2 La zone côtière du Gharb au Maroc .............................................................................. 15

3.2.3 La région de Nadhour en Tunisie ...........................................................................18

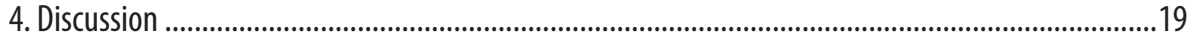

4.1 Précarité et empowerment: généralités et spécificités.....................................................19

4.2 Un nouveau regard sur le travail des ouvrières en agriculture .............................................20

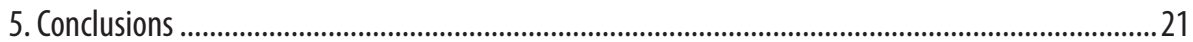

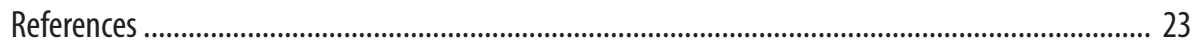




\section{Résumé}

En dépit d'une baisse remarquable de la main d'œuvre agricole d'une manière générale, l'effectif des femmes salariées dans le secteur agricole connaît une croissance substantielle liée particulièrement à l'intensification de l'activité agricole. En se centrant sur les problèmes d'accès des femmes à l'emploi, les travaux de recherche traitent peu ou rarement les conditions d'exercice du travail. Larticle porte sur les conditions de travail des femmes dans le secteur agricole à partir de l'analyse de trois cas d'étude dans trois pays de la région MENA: l'Ouest de Delta en Egypte, la zone côtière du Gharb au Maroc, et la région du Nadhour en Tunisie. Nous analysons dans quelle mesure le travail agricole pourrait constituer un moyen de précarité ou d'empowerment pour les femmes ouvrières. Nous avons montré que la précarité du travail agricole est multiforme dans les trois cas. Néanmoins, les ouvrières parviennent tout de même à contribuer à leur propre empowerment malgré la précarité associée aux conditions d'exercice du travail.

Mots clés: Empowerment, précarité, ouvrières, travail agricole, Egypte, Maroc, Tunisie

\section{Introduction}

Dans la plupart des pays en développement la contribution des femmes au travail agricole est incontestable (Bisiliat et Fieloux, 1992). Les femmes rurales constituent une force active importante pour la production agricole et contribuent au revenu familial à travers leurs activités agricoles et paraagricoles. En dépit d'une baisse générale de la main d'œuvre agricole, l'effectif des femmes salariées en agriculture connaît une croissance remarquable selon le rapport de la banque Mondiale (2009). Dans la région MENA, le rapport de la FAO (2005) met l'accent sur la féminisation notoire du travail agricole. En effet, les phénomènes migratoires et d'exode rural attirent de plus en plus les hommes alors que la participation féminine dans l'emploi agricole est en nette augmentation. Pendant que certains considèrent le travail des femmes en agriculture comme un espace d'exploitation où l'application de la législation de l'emploi est quasiment absente d'autres le voient comme un secteur dynamique qui fournit des opportunités de travail à petite échelle pour les populations pauvres en milieu rural (Cotela, 2002).

Pour les premiers, la division de la main-d'œuvre agricole entre les femmes et les hommes tend à confiner les femmes dans un rôle de 
producteur pour les besoins du ménage: non payée et non comptabilisée par les statistiques. La main-d'œuvre féminine n'est pratiquement pas reconnue dans le domaine de l'agriculture. L'étude de Ferchiou (1985) montre que le travail de la femme rurale en Tunisie est perçu comme le prolongement spontané et, en quelque sorte, obligé de ses responsabilités domestiques. En milieu rural marocain, la plupart des femmes rurales exerçant une activité à l'extérieur de leurs domiciles sont des ouvrières dans le secteur agricole ou agro-industriel. La catégorie professionnelle combine ici les deux occupations, ce qui ne permet pas de déterminer précisément les proportions (Damamme, 2005). Cependant, en vérifiant le statut professionnel et la branche d'activité, on peut dégager que le salariat agricole au Maroc emploie $58 \%$ des femmes rurales alors que le salariat en industrie emploie seulement I4\% (El Amrani, 2008). D'autre part, Le rapport de la Banque Mondiale (2008) souligne que $70 \%$ des pauvres de la région MENA vivent en milieu rural et dont la plupart appartiennent à certains groupes sociaux vulnérables tels que les ménages dirigés par les femmes, les sans-terres et les travailleurs agricoles. Dans ce sens et comme l'affirme Laroussi en Tunisie (2002), la femme ouvrière dans le secteur agricole serait doublement marginalisée de par son appartenance à deux groupes vulnérables : celui des femmes rurales et celui des travailleurs en agriculture. Dans son étude sur les ouvrières agricoles en Egypte, Kishk (1996) souligne que malgré la hausse remarquable en termes de participation des femmes rurales au travail salarié, les ouvrières demeurent la catégorie sociale la plus vulnérable en milieu rural et leurs situations se détériorent sans cesse.

Pour les seconds, l'emploi salarié en agriculture pourrait constituer au-delà de son précarité générale, un moyen pour renforcer les capacités des femmes. Selon Garnier et Guettat (2006), le travail agricole devrait à priori permettre aux femmes d'assurer une mobilité territoriale et de constituer une opportunité pour franchir le cadre familial et villageois. Shalaby (I99I) souligne que l'exploitation et la reconquête de nouveaux terrains en Egypte permettent un changement des activités traditionnelles des femmes rurales en leur offrant de nombreuses opportunités du travail rémunéré et un salaire plus élevé que celui appliqué dans la vallée du Nil. De ce point de vue, le travail constitue un moyen d'émancipation et d'amélioration de la situation socio- économique des femmes rurales, en leur permettant de répondre à leurs besoins, à ceux de leurs enfants sans passer par l'autorité de l'homme.

Par ailleurs, la résolution des problèmes sociaux des femmes par l'économie de marché découle d'une instrumentalisation des questions politiques liées aux droits des femmes. Cela mène à une vision 
réductionniste limitant tous les droits des femmes à un problème d'accès au travail et aussi tout le discours des Etats autour des droits des femmes et l'empowerment, à des activités génératrices de revenu sans pour autant analyser les circonstances et les conditions réelles d'exercice de ce travail (Kumar, 2004) En revanche, si le "travail des hommes" réfère plus visiblement aux conditions d'exercice de cette activité, l'approche du travail des "femmes" renvoie souvent à des comportements d'activités (elles travaillent ou non en emploi salarié) (Gollac, Volkoff, 2002). Dans la région MENA, la catégorie ouvrière reste oubliée par la recherche et le développement (Kheir, 2004). Dans la plupart des travaux existants la vision homogénéisante et parfois "victimisante" de la femme rurale masque une variabilité de profil, de statuts, de projets et d'identités et tend donc à percevoir les femmes ouvrières à travers une position d'acteurs passifs.

Le présent article s'attache à comprendre et à analyser les conditions de travail des femmes pour analyser dans quelle mesure le travail agricole pourrait constituer un moyen de précarité ou d'empowerment pour les femmes ouvrières. L'analyse porte sur trois régions agricoles: l'ouest de delta en Egypte, la zone côtière du Gharb au Maroc, et la région du Nadhour en Tunisie. Le papier est organisé de la façon suivante: une présentation du cadre d'analyse suivi d'une présentation de la méthodologie et des principaux résultats d'analyse qui seront présentés et discutés.

\section{Cadre d'analyse}

\subsection{Deux dimensions pour appréhender le travail de femmes en agriculture}

\subsubsection{Précarité}

Le rapport Wresinski en 1987 présente la définition de référence de la précarité 'la précarité est l'absence d'une ou plusieurs éléments de sécurité, notamment celle de l'emploi, permettant aux personnes et familles d'assumer leurs obligations professionnelles, familiales et sociales, et de jouir de leurs droits fondamentaux". La précarité touche donc des individus issus de tous les milieux sociaux ou professionnels mais affecte plus fortement les catégories les plus démunies. Ainsi, le concept de précarité se place souvent au centre des travaux consacrés aux situations des populations fragiles (femmes, enfants, émigrés, ...). Le travail des femmes est caractérisé par son universelle précarité, qui se décline au Sud 
par le travail informel et au Nord par le temps partiel auquel s'ajoutent les nouveaux contrats précaires. Si la précarité correspond à un état, la précarisation désigne le processus qui a aboutit à cette situation. La précarisation est un processus qui empêche l'accumulation qu'elle soit monétaire ou professionnelle. La précarisation sociale constitue un processus mettant les individus en situation de précarité et de maltraitance en les enfermant dans la pauvreté et la corvéabilité (Le blanc, 2006). Elle consiste donc en une déstructuration des droits sociaux, une exposition à la violence et une privation de l'accès à un minimum de capacitation et aux droits fondamentaux.

Dans cet article, nous voudrions mettre à l'épreuve la présomption d'une interaction entre une situation précaire et des sujets qui vivent cette situation, à la fois en termes de vulnérabilité ou de fragilisation mais aussi en termes d'adaptation mise en ouvre pour la surmonter ou du moins pour réduire son acuité. Notre analyse s'attache à caractériser les différentes expressions de la précarité auprès des ouvrières agricoles dans les trois contextes sans pour autant analyser la précarisation en tant que processus.

\subsubsection{Empowerment}

Le concept d'empowerment est un concept polysémique qui se prête à une compréhension pluraliste. Au plan individuel, Eisen (1994) définit l'empowerment comme la façon par laquelle l'individu développe ses habiletés favorisant l'estime de soi, la confiance en soi, l'initiative et le contrôle. Certains parlent de processus social de reconnaissance, de promotion et aussi de capacité à satisfaire leurs besoins, à régler leurs problèmes et à mobiliser les ressources nécessaires dans une visée de contrôle de leur propre vie (Gibson, I99I). L'empowerment comporte quatre composantes essentielles: la participation, la compétence, l'estime de soi et la conscience critique. Le processus d'empowerment est engagé lorsque ces quatre composantes sont en interaction. Ce processus proactif est centré sur les forces, les droits et les habiletés des individus et de la communauté, plutôt que sur les déficits ou les besoins (Gibson, I99I). L'empowerment touche le plus souvent des groupes de personnes sans pouvoir reconnu. Flaquet (2003) met l'accent sur le caractère spécifiquement féministe et du Sud de la notion d'empowerment. La notion d'empowerment est étroitement liée à celle du pouvoir. Pour les femmes, le pouvoir constitue un "pouvoir-capacité" qui serait permis par un meilleur contrôle sur les ressources matérielles et non matérielles, tout en élevant leur "estime d'elles-mêmes" (Moser, I989). Selon Drinkwater (2005), l'empowerment renvoie à un processus de changement dans une 
perspective d'amélioration du bien être physique, économique et social des femmes et dont la durabilité est tributaire de trois types de changement:

i. Un changement au niveau des individus où les femmes deviennent des acteurs capables d'analyser leurs propres situations et de prendre en main leurs propres actions et décisions.

ii. Un changement de structure affectant les structures familiales et les formes conventionnelles de pouvoir de façon à les faire durer.

iii. Un changement des relations par l'établissement de nouvelles relations avec d'autres acteurs sociaux, la formation de coalition et de soutien mutuel.

Ces relations permettent de développer des capacités de négociation de façon à devenir des acteurs de changement, contourner les structures pour faire valoir les droits, la dignité et améliorer les moyens de subsistance. Hofmann (2003) parle d'empowerment communautaire qui correspond au résultat de la participation à des actions politiques et collectives dans l'objectif d'une meilleure redistribution des ressources favorables pour le groupe.

\subsection{Méthodologie}

L'enquête par questionnaire a porté sur un échantillon de 150 ouvrières (50 dans chaque contexte). L'enquête ne prétend pas à une représentativité statistique. Néanmoins, les femmes enquêtées ont été choisies de façon à représenter autant que possible la diversité des situations existantes (âge, état matrimonial, profil, etc....). Lenquête a porté sur les axes suivants: identification de l'ouvrière et de sa famille, l'accès au travail, les conditions d'exercice, et la perception de l'emploi agricole. Une quarantaine d'entretiens ont été réalisés auprès des ouvrières et auprès des différents acteurs impliqués dans le circuit du travail agricole (les chefs d'exploitations, les gérants, les chefs d'ouvrières, les transporteurs, les membres de la famille, etc.). Nous avons réalisés des observations dans les exploitations agricoles à différents moments, à différents stades des cultures et dans différents lieux et moments (dans le village, les points de rassemblement des ouvrières, le moment du paiement...etc). Lobjectif n'étant pas de faire une comparaison entre les trois cas d'étude mais plutôt de mettre en lumière les conditions de travail des femmes dans les trois situations pour enrichir la réflexion sur ces trois pays, socialement, culturellement et historiquement assez proches (Hopkins, 198I) et qui se sont plus récemment engagés dans des réformes économiques et politiques avec beaucoup de similitudes (Assaad, 2006). 


\subsection{Présentation de la zone d'étude}

\subsubsection{Cas de l'Ouest de Delta en Egypte}

Zone à vocation principalement agricole, l'Ouest Delta en Egypte est constitué par des exploitations agricoles variant entre 50 et 300 feddans (I feddan équivaut $0,42 \mathrm{ha}$ ). Les cultures maraîchères et l'arboriculture constituent les principales cultures de cette zone. Lessentiel de la production est destinée à l'exportation. Ce type d'exploitation fait appel à une main d'œuvre agricole principalement féminine en provenance des villages avoisinants le Delta. Le revenu issu de ce travail permet à ces ouvrières de contribuer au revenu familial, souvent très restreint. Par ailleurs la mise en vigueur de la loi $n^{\circ} 96$ de 1992, des réformes foncières entre propriétaires et locataires dans la région de Delta a transformé plus de 440000 petits paysans en ouvriers agricoles et par conséquent a aboutit à l'accroissement de la demande en main d'œuvre agricole dans la zone.

\subsubsection{Cas de la zone côtière du Gharb au Maroc}

La zone côtière du Gharb est située sur la côte atlantique au Nord ouest du Maroc. La disponibilité des terres agricoles (essentiellement de statut collectif), des eaux souterraines et aussi de la main d'œuvre agricole a transformé la zone depuis une trentaine d'années en un pôle d'attractivité pour les investisseurs espagnols au départ et marocains par la suite. Lintensification agricole a été permise par la forte utilisation des intrants agricoles et par l'introduction des nouvelles techniques agricoles (l'irrigation localisée, les abris serres, ...). Lintroduction des cultures maraîchères exigeantes en travail a eu pour première conséquence la création d'une forte demande en main d'œuvre agricole. Les producteurs de la culture de fraise, font appel à une main d'œuvre principalement féminine. Ce choix s'explique par des qualités féminines supposées telles qu'une plus grande docilité ou une grande capacité de travail. Certaines ouvrières sont désignées pour travailler d'une manière saisonnière, dans les exploitations de fraises dans la région de Huelva au sud de l'Espagne dans le cadre des contrats d'origine ${ }^{\mathrm{I}}$. Entre 2007 et 2008, environ I2.000 femmes marocaines ont été sélectionnées. Ce chiffre a atteint près de I6.000 personnes au cours de la saison 2008/2009.

\footnotetext{
1 Les contrats saisonniers d'origine: contrats qui donnent lieu à une autorisation de travail et de séjour à durée déterminée (entre trois et neuf mois par an) qui sont liés à une zone géographique, à une profession et à un employeur spécifique. Ce type de contrat est destinée aux femmes mariées âgées de 20 à 40 ans, travaillant en zone rurale et ayant des liens familiaux (enfants) qui exigeront un retour au pays à la fin de la saison agricole conformément à l'engagement signé au départ.
} 


\subsubsection{Cas de la délégation de Nadhour en Tunisie}

La délégation de Nadhour fait partie du gouvernorat de Zaghouan qui se situe au nord de la Tunisie. Cette zone est caractérisée par l'importance de la main d'œuvre féminine au niveau des exploitations agricoles. Au cours des cinq dernières années, la zone a connu une dynamique économique importante suite à l'installation des activités industrielles implantées dans les zones rurales. L'activité industrielle contribue à la création d'au moins l'équivalent de mille postes d'emploi permanant et un nombre important d'emploi saisonnier. Ce pôle industriel est orienté essentiellement vers des activités non complémentaires à l'agriculture. Les opportunités offertes en matière d'emploi dans ces activités industrielles (une situation plus stable, un travail moins pénible, et une rémunération plus sûre) causent une vraie concurrence entre l'agriculture et l'industrie sur la main d'œuvre féminine. Dans ce contexte de concurrence, il ya des différents types de mobilités (sociales, professionnelles et géographiques) de la main d'œuvre agricole.

\section{Résultats}

\subsection{Contexte et conditions du travail: Précarité générale dansles trois contextes}

\subsubsection{La région de l'ouest de Delta en Egypte}

La personne chargée de la main d'œuvre agricole est un acteur clé dans l'ouest de Delta de l'Egypte appelé localement "entrepreneur". Celui-ci s'occupe de la recherche des ouvrières, de garantir le transport à partir des régions voisines ( $82 \%$ d'El-Monofia et $18 \%$ d'El Beheyra) jusqu'aux exploitations agricoles. A l'entrée de chaque village, les ouvrières se regroupent en attendant l'arrivée du camion collectif de l'entrepreneur qui les amènent jusqu'au "El-Mafreq"2. A ce point fixe de rassemblement de la main d'œuvre, les ouvrières reprennent un deuxième moyen de transport pour se rendre aux exploitations. En général, un minibus d'une capacité de I2 personnes, transporte 25 ouvrières.

"Fe me sens étouffée dans ce minibus, jarrive à peine à respirer, nous sommes serrées comme dans une boîte de sardines. Le camion reste quand même mieux que le minibus..." (Ouvrière, I9 ans)

2 El- Maferq: c'est un point fixe de regroupement des ouvrières venant de plusieurs villages avant d'être affectées en groupes à des exploitations agricoles. Au Maroc cet endroit est communément appelé Maouquef 
Arrivées aux exploitations vers $8 \mathrm{~h} 30$ min, la journée du travail commence à $9 \mathrm{~h}$ du matin et se termine à $\mathrm{I} 7 \mathrm{~h}$. Les femmes sont chargées des tâches liées à la plantation, le repiquage, l'épandage du fumier, la fertilisation et la cueillette. Durant les dernières années, les ouvrières exercent des tâches qui étaient réservées aux hommes tels que la taille des arbres et le transport des produits agricoles. Cependant, l'expérience de l'ouvrière, son savoir-faire n'implique pas une rémunération supplémentaire mais lui garantit plus de journées de travail par an.

Dans cette zone, les femmes enquêtées sont pour la plupart célibataires $(95 \%)$ avec $5 \%$ des divorcées. La moyenne d'âge est de I9 ans et celle à la première expérience du travail est de io ans. Labsence des ouvrières mariées se justifie par un contexte social qui méprise la mobilité et le travail agricole pour les femmes mariées. L'entrepreneur chargé du paiement des ouvrières prélève $40 \%$ du salaire net attribué aux ouvrières qui est de l'ordre de 25 livre égyptien (3.50 euro). Ainsi, $60 \%$ des enquêtées déclarent recevoir un salaire médiocre. $55 \%$ des femmes insistent sur la pénibilité du travail agricole. En effet, $80 \%$ d'entre elles ont été confrontés à des problèmes de santé liés principalement à la respiration, et aussi à des problèmes dermatologiques dus aux coups du soleil et à l'usage des produits chimiques. Selon les interviewées la relation entre l'entrepreneur et les ouvrières est souvent marquée par la violence physique et morale. En cas de faute jugée grave par l'entrepreneur (parler au moment du travail, se plaindre d'une fatigue,...), celui-ci se donne le droit de les priver de prendre la pause pour manger. Cependant, l'entrepreneur détient une valeur morale auprès de la famille de l'ouvrière. Il a la responsabilité de contrôler la fille depuis sa sortie du village jusqu'à son retour au foyer grâce au pouvoir que lui accorde la famille.

\subsubsection{La zone côtière du Gharb au Maroc}

Dans les exploitations de production de fraises de la zone côtière du Gharb, la durée moyenne du travail est de 8 heures à laquelle s'ajoute la durée du transport si l'exploitation est distante des villages des ouvrières. La durée du transport est variable et peut atteindre 2 heures. Les ouvrières ont droit à une pause d'une heure pour le déjeuner. La rémunération journalière du travail est entre 45 et 50 dirhams (environ 4,5 euro) reçu directement par l'ouvrière et payé par quinzaine. Le cas échéant, celles-ci reçoivent un montant supplémentaire de io drh pour les frais du transport. D'une manière générale, l'emploi dans les exploitations de production de fraise implique en majeure partie une main d'œuvre en provenance des villages limitrophes des villages. D'une part, les producteurs de fraises 
ont tendance à choisir une main d'œuvre dans les villages proches des exploitations pour minimiser le coût du transport et tâchent à garder quasiment les mêmes ouvrières pour assurer leur présence en période de pointe. D'autre part, les familles des ouvrières préfèrent que cellesci aillent travailler dans les fermes avoisinantes en compagnie d'autres ouvrières de la même famille (mère, cousines, belle sœur). Par ailleurs, il existe un autre domaine de travail pour les autres cultures (tomate, poivron, pomme de terre, pastèque,...) et pour des ouvrières en provenance d'autres localités qui se rassemblent chaque matin au niveau du Moquef (équivalent de Mafraq dans l'ouest de Delta) où les exploitants viennent les chercher. Pour la culture de fraise, le recours aux ouvrières du Moquef se fait uniquement en période de cueillette ou pour le travail à l'usine qui nécessitent une main d'œuvre plus nombreuse.

Au niveau de l'exploitation, le tâches à effectuer varient suivant le stade de la culture et suivent une division sexuelle du travail. En général, les femmes s'occupent, de la plantation, du désherbage et de la cueillette, tandis que les hommes se chargent de la fertilisation, du traitement phytosanitaire et du ramassage des caisses. Par ailleurs, le paillage plastique peut impliquer les deux sexes. $25 \%$ des ouvrières enquêtées travaillent aussi d'une manière saisonnière au niveau de l'usine pour l'emballage et le conditionnement des fruits. A l'usine, les ouvrières sont payées à l'heure (Io dirhams par heure) ou à la tâche et ont généralement accès à la caisse de sécurité sociale, quoique les conditions soient largement contestées par les ouvrières.

La rémunération est jugée faible par $75 \%$ des ouvrières vue que les opérations culturales du fraisier sont particulièrement pénibles et pouvant présenter des risques sanitaires (douleurs dorsales, la migraine, des problèmes dermatologiques, des problèmes de respiration et parfois des risques d'avortement). $69 \%$ des enquêtées déclarent avoir déjà rencontré un problème de santé lors de l'exercice du travail. Le salariat féminin en agriculture occupe une place importante dans le revenu du ménage. Pour $39 \%$ des femmes enquêtées, la contribution peut aller du quart à la moitié du revenu familial. Pour deux ouvrières enquêtées dont une divorcée et l'autre veuve, le travail agricole constitue l'unique source du revenu pour elles et pour leurs enfants. En revanche, ${ }_{2} \%$ des ouvrières disent ne pas contribuer au revenu du ménage. Il s'agit particulièrement d'ouvrières célibataires dont les familles disposent d'autres sources de revenu. Plus généralement, la contribution de l'ouvrière s'insère dans un système marqué par la pluriactivité où 2 à 5 membres de la famille sont actifs (la taille du ménage étant de 8 individus en moyenne). D'autre part la contribution de la femme ouvrière requiert souvent un caractère multiforme en tenant 
compte de son rôle important à la maison, pour l'éducation des enfants et aussi dans l'exercice d'autres activités non agricole (tapisserie, couture,...).

Par ailleurs, $19 \%$ des ouvrières déplorent le comportement autoritaire et parfois humiliant et agressif du chef d'ouvrier (surtout quand c'est un homme) et du gérant de l'exploitation. L'harcèlement, l'absence de sanitaires au niveau des exploitations accentuent la pénibilité des conditions de travail. En outre, l'implantation de l'agriculture intensive dans la zone et le recours important à la main d'œuvre féminine s'est traduit par un engouement sur le travail auprès des femmes y compris les jeunes filles en âge de scolarisation. Le corollaire est l'augmentation du taux d'analphabétisme du à la déperdition scolaire $(92 \%$ des enquêtées sont analphabètes) et du pourcentage de divorce. En effet, de nombreuses ouvrière se marient souvent très jeunes avec des ouvriers rencontrés à l'exploitation et finissent dans de nombreux cas par divorcer. A l'instar du travail dans le secteur informel, ce type d'activité demeure précaire de par l'absence totale des droits sociaux (assurance, retraite,...) au niveau des exploitations agricoles. Aussi, aucune des ouvrières enquêtées n'adhère à une association ou à un syndicat du travail.

\subsubsection{La région de Nadhour en Tunisie}

Dans un contexte de concurrence entre l'industrie et l'agriculture sur la main d'œuvre féminine, la zone de Nadhour en Tunisie est caractérisée par une mobilité de la main d'œuvre agricole provenant des zones voisines. L'échantillonnage de Nadhour se caractérise par une taille de ménage importante (6 personnes/ménages), mais la taille de ménage ne détermine pas forcément la taille de la force de travail. En effet $35 \%$ des femmes enquêtées contribuent à plus de $50 \%$ du revenu familial, dont $20 \%$ contribuent à I00\% de ce revenu. Ces femmes ouvrières étaient toujours obligées de travailler avec des salaires dérisoires par besoin de survie. Notamment $2 \%$ des femmes peuvent épargner. A l'échelle de l'exploitation, les femmes ouvrières sont plus présentes que les hommes, ce qui peut être expliqué par un critère de recrutement de la main d'œuvre basé principalement sur le coût ( 5 Dinars/J pour la femme et $6 \mathrm{DT} / \mathrm{J}$ pour l'homme) et sur la disponibilité. Elles travaillent en moyenne de 9 àıo heures par jour. A l'échelle de l'exploitation, elles s'occupent des tâches manuelles liées au semis, sarclage, binage, fertilisation, irrigation, récolte, alors que les hommes sont responsables du labour et du portage et du transport des produits agricoles.

Les femmes ouvrières ont toujours mené un travail diversifié, éparpillé entre plusieurs espaces et périodes. Ce travail présente des répercussions 
négatives à l'échelle de l'exploitation. Ces effets sont dus à la pénibilité du travail agricole selon $90 \%$ des ouvrières enquêtées à l'origine des problèmes de santé pour $38 \%$ parmi elles (I $9 \%$ présentent des problèmes respiratoires, $2 \%$ avortement et $15 \%$ des brûlures suite à l'usage des produits phytosanitaires). D'autre part, les ouvrières sont exposées au risque d'harcèlement moral et sexuel de la part du chef d'exploitation ( $15 \%)$, à l'éloignement $(48 \%)$ du lieu du travail, et à la violence conjugale à cause du retour tardif du travail (I $2 \%)$.

En outre, les femmes assument à côté de leurs tâches dans l'exploitation des tâches domestiques. Elles contribuent à une production domestique non marchande qui comprend le ménage, la cuisine, l'éducation des enfants, l'entretien des relations sociales, la recherche de l'eau et du bois du chauffage. Elles sont également très actives dans la transformation et la conservation des produits végétaux et animaux, qui sont destinés surtout à la consommation familiale. Elles mènent par ailleurs des activités d'artisanat pour améliorer le revenu de leur famille: tissage, vannerie, poterie...Ces responsabilités ont évolué et se sont multipliées surtout avec la désagrégation de la famille élargie et la diminution de l'entraide féminine traditionnelle (grands mères, tantes, ...).

“... En effet, il est rare de voir des femmes assises à ne rien faire, lorsque nous nous rassemblons pour bavarder et chaque fois que nous avons un moment libre, nous tissons. D'ailleurs, les couvertures et les tapis constituent une véritable épargne, car chaque fois quil a besoin d'argent liquide, le chef de famille peut aller les vendre au marché hebdomadaire le plus proche et avoir recours à la somme que ces produits lui rapportent..."

Limportance des responsabilités domestiques, la présence d'un contexte socioculturel lourd et la faiblesse des organismes d'appui en faveur des femmes les privent d'avoir un accès aux ressources matérielles et aussi non matérielles (information, formation, conseil et vulgarisation).

\subsection{Quelques éléments de l'empowerment des ouvrières}

\subsubsection{La région de l'ouest de Delta en Egypte}

Laugmentation des salaires des femmes qui travaillent de façon constante au cours de cette période a été suivie par une diminution de la différence entre les deux salaires hommes et femmes travaillant dans ces fermes. Ce qui peut améliorer la situation des femmes au sein du ménage. 
"Fe travaille dans les fermes depuis plus de 25 ans, le travail était toujours le même, les jours se ressemblent, mais concernant le salaire journalier, jai remarqué que depuis les années 1990 le salaire des ouvrières était de 5LE, en 1995 il est passé à IoLE, 2000 il est devenu $20 L E$ et maintenant il est de $25 \mathrm{LE}$. Cependant la différence entre le salaire des ouvrières et celui des ouvriers a diminué...", gérant d'une exploitation (50 ans).

Par ailleurs, le travail au niveau des fermes a modifié l'organisation traditionnelle du travail. Les femmes sont plus présentes dans l'exploitation, elles assument des tâches jadis consacrées aux hommes, et leurs salaires est équivalent à celui des hommes. Cette nouvelle organisation permet une émergence d'une forme d'empowerment des femmes. Aussi, le travail agricole permet de franchir le cadre villageois, de diminuer les charges domestiques, de préparer son trousseau de mariage et aussi de choisir son mari.

“.... Fe travaille pour préparer mon mariage. Fe participe à une tontine pour se procurer la vaisselle, des vêtements et des équipements de cuisine pour mon mariage. Chaque mois, je me rend au marché du village pour se procurer d'une partie des achats", ouvrière (I8 ans).

Le fait d'avoir un salaire lui donne plus d'autonomie et d'un estime de soi, en effet l'intérêt accordé à l'épargne est signalé par 30\% des enquêtées. D'autres contribuent au financement de leurs scolarisations. Le travail constitue ainsi une opportunité pour ses filles de continuer leurs études et d'une manière générale de réduire le taux d'analphabétisme “...pendant l'été, je recrute des filles, même des étudiantes des universités qui soubaitent financer leurs études,... Ce n'était jamais une honte de travailler dans des fermes..." entrepreneur (54 ans).

\subsubsection{La zone côtière du Gharb au Maroc}

Lautonomie financière, les différents usages du revenu et l'estime du soi sont autant de facteurs d'empowerment lié au travail des femmes saisonnières en agriculture. I2\% des ouvrières affirment que le revenu du travail est en totalité utilisé pour subvenir à leurs besoins personnels (vêtements, bijoux,...). Ce revenu peut également servir d'appui pour préparer à leurs mariages comme pour cette jeune ouvrière de 19 ans: "F'ai travaillé pour une période à l'usine, ça m’a permis d'épargner ıoooo dirhams (équivalent de 900 euros) que je compte utiliser pour acheter des bijoux et des meubles pour mon mariage, l'été prochain". 
Quant aux femmes mariées, le travail agricole permet à la femme d'être en totalité ou en partie indépendante de son mari et d'acquérir une autonomie financière. La rémunération du travail agricole constitue une opportunité pour échapper aux tâches domestiques souvent pénibles et non rémunérées surtout pour les jeunes filles. "Mon père préfêre que je travaille seulement pour notre agriculture, et ma mère m'envoie chaque jour pour chercher le bois du feu dans la forêt, c'est un travail pénible mais sans argent. Franchement je préfere aller travailler avec mes copines dans les fermes" ouvrière (I5 ans).

En plus de cette dimension économique liée à l'emploi agricole, une dimension symbolique peut lui être attribuée, en particulier une augmentation de la confiance et de l'estime du soi auprès des ouvrières. Le recours à une main d'œuvre essentiellement féminine et la désignation spécifique des femmes pour les contrats d'origines pour travailler en Espagne constitue une forme de reconnaissance de la place de la femme dans l'agriculture et aussi de son savoir-faire. Au niveau local, la culture $\mathrm{du}$ fraisier est reconnue comme une culture des femmes "Les garçons ne savent pas travailler dans la culture du fraisier, Ce sont les femmes qui maîtrisent parfaitement sa conduite technique depuis la plantation jusqu'à la cueillette et le conditionnement" (une ouvrière, 25 ans). Si ce choix augmente l'estime du soi auprès des femmes, les hommes expriment une certaine envie ou déception "Dans cette région, les femmes ne chôment pas, elles sont partout dans les exploitations du fraisier, elles vont même jusqu'à travailler en Espagne. Les hommes restent à la maison ou vont au Moquef, ils n'ont pas du tout les mêmes opportunités", a indiqué le frère d'une ouvrière (25 ans).

On remarque aussi l'émergence de nouveaux métiers. La femme ouvrière peut accéder au rang du chef d'ouvrières (ou caporale) qui s'occupe de la recherche des ouvrières dans les villages, de la supervision et le contrôle du travail au niveau de l'exploitation, la comptabilisation des heures de travail, etc. Elle joue le rôle d'intermédiaire entre le gérant de l'exploitation et les ouvrières. Par ailleurs, le statut de la femme chef d'ouvrière est en luimême une source de reconnaissance de ses capacités de gestion et de ses compétences. Cette reconnaissance a un impact visible sur les rapports de genre, l'entourage familial et villageois de la femme: "Mon mari minterdisait de travailler dans l'agriculture malgré que nous étions pauvres, je suis allée deux fois en cachette pour travailler, le gérant a constaté mon intelligence et mes capacités de calculer rapidement le nombre de caisses et des barquettes de fraises. Il má désigné comme chef d'ouvrière. F'ai raconté l'histoire à mon mari et depuis il était fière de moi, son respect a augmenté et il ma autorisé de travailler" (chef d'ouvrières, 39 ans) 
Le travail en agriculture implique un changement au niveau des relations qu'entretient la femme avec son entourage familial et aussi au niveau de l'exploitation. Le choix des exploitations se fait au sein de groupes d'affinité regroupant des ouvrières ayant des liens familiaux, de voisinage ou de copinage. En effet, les ouvrières se déplacent en groupe pour travailler dans les mêmes exploitations, elles partagent le repas ensemble pendant la pause, discutent de leurs problèmes, chantent et rentrent ensemble au douar en fin de journée. Ces groupes permettent de créer une certaine convivialité pendant le travail mais constitue également une sorte de contrôle social et d'assurance pour les familles des ouvrières (par exemple le cas où la maman travaille avec ces filles au niveau de la même exploitation). Comme dans l'ouest de delta, la tontine est une pratique très courante auprès de ces groupes d'ouvrières. Elle constitue un moyen pour préparer au mariage, acheter des bijoux ou des vêtements. D'autre part, le revenu agricole procure pour certaines ouvrières $(9 \%)$ une assurance financière pour octroyer un microcrédit en vue de l'investir dans l'agriculture ou dans l'élevage ou bien pour participer à la construction des maisons en dur et aux frais de mariages des enfants ... etc. Le lieu de travail s'apparente ainsi à un moyen d'apprentissage, d'interconnaissance et d'échange d'expériences. De nombreuses ouvrières ont rencontré leurs maris lors de l'exercice du travail. "Fe suis contente de mon travail, il ma permis de rencontrer mon mari qui était chef d'ouvrier et de fonder une famille, d'acquérir quelques bijoux. Maintenant je ne travaille plus dans les fermes, je travaille uniquement à l'usine durant trois mois par an pour aider mon mari et subvenir au besoin de mes enfants", a dit une ouvrière, âgée de 24 ans, mariée et a deux enfants.

Pour certaines femmes, le travail constitue une opportunité pour franchir le cadre familial et villageois en leur assurant une certaine mobilité. En effet, l'intensification agricole et le recours à la main d'œuvre féminine a aboutit à une légitimation progressive et une acceptation sociale du travail des femmes en agriculture. Dans d'autres villages de la région qui n'ont pas connu les mêmes dynamiques agraires, l'emploi salarié des femmes dans l'agriculture demeure très mal perçu socialement. Ainsi, l'accès au travail (indépendamment de sa nature et de ces conditions) constitue en lui-même une sorte d'empowerment.

Le désir de la mobilité s'accompagne d'un espoir d'atteindre une vie et un avenir meilleur pour les ouvrières qui vont travailler en Espagne “ F'ai toujours rêvé de voyager, de partir en Europe, maintenant, je suis divorcée avec deux enfants à ma charge et jai une seule envie c'est d'être sélectionnée pour les contrats d'origine pour sortir de ma souffrance quotidienne au sein de la famille et dans le village et de sauver mes enfants" (ouvrière,19 ans). 
Pour certaines ouvrières les contrats d'origine pourrait constituer une voie légale pour traverser les frontières vers l'Europe. Selon Hellio (2008), le taux de "fugues" des travailleuses marocaines avait atteint $50 \%$ en 2006.

\subsubsection{La région de Nadhour en Tunisie}

Le revenu des femmes provient essentiellement du travail agricole et de la transformation des produits agricoles. Elles travaillent pour compléter le revenu du chef de ménage ou bien pour combler l'insuffisance de son salaire et pour pouvoir se procurer ce que le revenu du chef de ménage ne permet pas. En effet, les revenus obtenus permettent à la femme de répondre à de nombreux besoins de la famille. Elles dépensent pour la maison, les enfants (santé, fournitures scolaires, nourriture, habillements, mariage) et elles dépensent relativement moins pour elles (achat des bijoux) "...je ne gagne pas beaucoup, mais je suis contente, ce travail me permet d'aider mon mari, fournir des besoins pour mes enfants...je me sens utile et autonome..." (ouvrière, 35 ans). Nous signalons que les femmes ouvrières se déplacent en groupe, elles sont de la même famille et rarement des voisines. En effet, pour $65 \%$ d'entre elles, le fait d'être active pour une femme est un atout considérable qui lui confère plus de liberté, un revenu, une certaine autonomie, une possibilité de contacts multiples, un apprentissage d'une action et une participation à la décision à l'échelle de son foyer. $92 \%$ des femmes enquêtées se réunissent entre elles de façon informelle, en attendant la camionnette du chef d'exploitation à côté de l'épicier ou de la fontaine publique. Au moment de la pause du repas, elles partagent des discussions jalonnées d'humour et aussi chargées des problèmes. D'où ce travail peut avoir des répercussions sur la confiance des femmes en elles-mêmes, peut aussi émerger de nouveaux aspects identitaires tout en modifiant des structures et des règles sociales classiques. La concurrence entre le secteur agricole et industriel sur la main d'œuvre féminine a des effets sur la relation entre les ouvrières agricoles et l'exploitant. Dans ce sens les ouvrières peuvent intervenir dans le choix des chefs d'exploitation et réciproquement les chefs d'exploitation mettent en ouvre plusieurs stratégies pour faire face à un manque de la force du travail féminine. Ils essayent de créer un climat de confiance et de compréhension (paiement par jour ou par semaine, flexibilité en cas d'absence ou de maladie, usage de moyen de transport...). 


\section{Discussion}

\subsection{Précarité et empowerment: généralités et spécificités}

La durée du travail, la faible rémunération, la quasi absence des droits sociaux, et la pénibilité des conditions du travail constituent autant de facteurs de précarité de l'emploi agricole des femmes dans les trois cas d'étude. En revanche, la précarité prend une diversité de formes selon les contextes. Lorganisation du travail dans l'ouest de Delta en Egypte constitue un élément de précarisation visible. Le circuit du travail piloté par l'entrepreneur est souvent caractérisé par la violence, par des rapports de domination et par de l'exploitation. Ce circuit étant accepté socialement, les ouvrières travaillant en dehors de ce circuit sont mal vues. Dans le cas marocain, l'histoire de l'introduction de la culture du fraisier dans la zone a conduit à une légitimation sociale progressive du travail des femmes spécifiquement dans ce secteur et à une hiérarchisation sociale du travail. Le travail au Moquef, quoique mal perçu et parfois lié à des activités déshonorantes ou peu correctes, reste mieux rémunéré (70 drh/ jour, environ 7euros/ jour) par rapport au travail dans les fermes du fraisier (4,5 euros/jour). Un des indicateurs symboliques de cette différenciation est que les ouvrières au Moquef travaillent le visage caché contrairement aux ouvrières dans les exploitations du fraisier qui travaillent le visage découvert. En Tunisie, la rémunération du travail agricole des femmes peut passer par un des membres de sa famille. Le paiement indirect des ouvrières renvoie au rapport patriarcal qui peut être lié à ce type d'activité. D'autre part, la rémunération des femmes parfois plus faible à celle des hommes, marginalise la femme par rapport au processus de développement et au système de profit. En revanche, certaines opportunités peuvent être perçues à priori comme des facteurs susceptibles d'aboutir à un renforcement des capacités des ouvrières alors que sur le fond cela pourrait indiquer une précarité invisible. Le cas des contrats d'origine en Espagne véhicule sous l'argument déclaré d'un développement entre le Maroc et l'Espagne, une "légalité de façade des contrats qui crée l'illégalité de fait sur le terrain" (Brelan, 2008). Dans ce contexte, l'organisation de la production et du rendement et la coopération patronale s'apparente largement à une organisation industrielle. Pour ce type d'agriculture, la main d'œuvre constitue la seule variable d'ajustement moyennant la réduction des coûts salariaux pour faire face à la concurrence internationale et à la demande élevé en main d'œuvre surtout en période de cueillette (Toronjo, 2008). 
Les ambitions et les espoirs qu'attachent les saisonnières en Espagne au travail en Europe peuvent se heurter à la pénibilité du travail en agriculture intensive et à la précarité des conditions. Sayad (I999) a fait référence à cette dynamique par "là où il y a des tâches dévalorisées, comme dans le travail agricole, il y a de la main-d'œuvre étrangère”.

Malgré le diagnostic d'une précarité générale et spécifique au niveau des études de cas, plusieurs indicateurs d'empowerment peuvent être associés au travail des ouvrières. L'autonomie financière, l'estime du soi, la mobilité, l'accès au travail, le partage d'expériences au sein des exploitations, la contribution au besoin du ménage, la préparation au mariage constituent les principaux indices d'empowerment pour les ouvrières. Par ailleurs, en Egypte le travail agricole se caractérise par une dimension conjoncturelle liée à son exercice. En effet, le travail implique essentiellement les jeunes filles célibataires, qui souvent travaillent pour aider leurs familles, financer leurs études et préparer leurs mariages. Dès qu'elles se marient, elles s'arrêtent systématiquement de travailler. La durée du travail pour une femme est ainsi centrée sur une étape de son parcours. Au Maroc, les ouvrières dans les exploitations du fraisier peuvent, selon le cas, s'arrêter de travailler ou non après le mariage mais la faiblesse du revenu familial les astreignent, le plus souvent, à continuer le travail même après le mariage. Cette continuité est favorisée par la reconnaissance sociale de ce type du travail d'une part, et par une préférence des femmes mariées et ayant des enfants pour les contrats d'origine d'autre part. En Tunisie, le statut matrimonial de la femme n'interroge pas son droit au travail qui est lié beaucoup plus aux conditions socio-économique de la famille. D'autre part, la concurrence entre l'industrie et l'agriculture dans la région de Nadhour en Tunisie donne aux femmes une marge pour changer l'exploitation en cas de problèmes, et oblige les propriétaires à être plus flexibles et à mettre en place certaines facilités pour maintenir la disponibilité des ouvrières pendant les périodes de pointe.

\subsection{Un nouveau regard sur le travail des ouvrières en agriculture}

Si la catégorie ouvrière semble la plus sacrifiée de la recherche et du développement, l'analyse des conditions du travail des femmes indique une variabilité de profil, de statuts, de projets et d'aspirations liés à l'exercice de cette activité. Nombreux sont les indicateurs de l'hétérogénéité de ce groupe social souvent vu par un regard homogénéisant: Le statut matrimonial, l'âge, le revenu familial, les trajectoires des femmes, leurs positions (ouvrières, chef d'ouvrière, ouvrières dans le cadre d'un contrat d'origine, reçoit directement ou indirectement son travail,...etc.) et le 
contexte socio-économique du travail témoignent de la diversité de ce groupe. Dans ce sens Jacquet (I995) affirme : "Un de ses points de départ est de briser le mythe de l'homogénéité de la condition féminine et d'affirmer qu'à l'intérieur du groupe de femmes, il y a des différences de statut, des rapports de force et même de domination".

Par ailleurs, les éléments d'empowerment repérés (estime de soi, accès à la scolarisation, la préparation au mariage, et la mobilité) montrent l'importance de l'empowerment crée au niveau individuel. Ce type d'empowerment renseigne sur un processus d'autonomisation développé par les ouvrières pour faire face à leurs contraintes et pour améliorer leurs conditions souvent précaires. Ces capacités et initiatives individuelles sont rendues, le plus souvent, invisibles par la vision "victimisante" attachée au travail des ouvrières en agriculture. Néanmoins, au-delà du niveau individuel, le changement n'atteint pas le niveau structurel et relationnel au sens de Drinkwater (2005) ni le niveau communautaire (Hofmann, 2003). Dans les trois contextes, les structures familiales et les formes conventionnelles du pouvoir (relation patriarcale, domination, etc.) continuent à façonner le travail agricole. La faiblesse des syndicats ne s'est pas compensée par le développement des mobilisations ou des organisations des ouvrières pour faire valoir leurs droits. Ce repli de l'empowerment au niveau individuel remet en cause la capacité des femmes à transformer leurs situations et à aboutir à des transformations profondes de leurs conditions de travail. Par ailleurs, nous nous interrogeons autour du type d'appui et d'accompagnement à apporter à ce groupe longuement marginalisé.

\section{Conclusions}

Les trois études de cas présentent des similarités et des divergences dans les conditions et l'organisation du travail des femmes en agriculture. Le travail agricole est caractérisé généralement par la précarité de ses conditions (faible rémunération, exploitation, absence de sécurité sociale,...), par des rapports hiérarchiques et violentes au sein de l'exploitation et par une perception sociale négative vis-à-vis de la femme ouvrière. Cependant, la précarité de la situation n'exclue pas la capacité de la femme ouvrière à s'adapter et à contribuer à son propre empowerment (self-empowerment). Cela passe principalement par le développement de la confiance, l'estime de soi, une certaine autonomie financière, et par l'accès à l'éducation. Cet empowerment n'est pourtant pas suffisant pour apporter un grand changement sur la situation générale des ouvrières ni 
sur le processus de précarisation associé au travail. Il reste en effet un "Empowerment précaire" et loin de faire face aux enjeux du marché et surtout au développement d'une agriculture capitaliste, de plus en plus demandeuse de la main d'œuvre féminine. Cette situation est aggravée par le fait que les fluctuations du marché international rendent la main d'œuvre le facteur le plus susceptible pour une réduction des coûts. Ainsi, l'intervention de l'Etat et de la société civile est susceptible de contribuer à une amélioration effective des conditions de travail des femmes dans les exploitations agricoles et de renforcer l'empowerment au-delà du niveau individuel vers des aspects plus structurels et relationnels. Cela passerait par le renforcement du cadre légal du travail agricole, des droits sociaux et économiques et aussi des syndicats agricoles. La recherche aurait un rôle à jouer dans la mise en évidence des conditions de travail de ces acteurs invisibles et oubliés par la recherche et le développement et dont les droits sont bafoués sous le silence des syndicats du travail et l'absence de règles et lois régissant ce type d'emploi. 


\section{References}

Assaad R., (2006), "Why Did Economic Liberalization Lead to Feminization of the Labor Force in Morocco and De-feminization in Egypt in Globalization and Gender?" Economic Participation of Arab Women", Cawtar, Tunis.

Banque Mondiale, (2008). Note sur l'agriculture et le développement rural au Moyen-Orient et en Afrique du Nord, URL: http://go.worldbank. org/KV7 $\mathrm{ABQI}{ }_{7}$ So.

Bisilliat J., Fieloux M. (1992). "Femmes du Tiers monde. Travail et 2uotidien". LHarmattan, Paris, p.I22

Cotula L., (2002). "Gender and Law - Women's Rights in Agriculture”, FAO, Rome.

Damamme A., (2005). "Le genre à l'épreuve du développement au Maroc: Discours et pratiques concernant la place des femmes dans les projets", Thèse en géographie à l'université d'Orléans.

Drinkwater, Michael. (2006). "L'Empowerment des Femmes au Bangladesh: L'Histoire Émergente de la ESI..”.

Étude de Cas écrite pour le CSPS-CARE.

Échange d'Information sur les méthodes de recherche sur l'empowerment des femmes. Juillet I4-15, 2006. Emory University, Atlanta, Georgia, USA.

EISEN,A. (1994). "Survey ofneighbourhood-based, comprehensive community empowerment initiatives", Health Education Quarterly, vol. 2I, No 2, 235-252.

El Amrani M., (2008). "Développement des Indicateurs Sensibles au Genre dans la Base de Donnée AQUASTAT, cas du Maroc,". Rapport FAO et CAWTAR.

Falquet Jules, (2003). "Femmes, féminisme et développement", In Bisilliat Jeanne, "Regards de femmes sur la globalisation", Karthala.

FAO, (2005). Gender and Law: "Women's Rights In Agriculture"; FAO Legislative, Study 76. Rev. I, URL: http://www.fao.org/docrep/o05/Y43IIE/ y43Ireo5.htm\#TopOfPage

Ferchiou S., (1985). "Les femmes dans l'agriculture tunisienne". Edisud.

GIBSON C.H., (r991). "A concept analysis of empowerment". Journal of Advanced Nursing, I6, 354-36r.

Gollac Michel, Volkoff Serge, (2002). "La Mise au travail des stéréotypes de genre: les conditions de travail des ouvrières", Travail, genre et sociétés, $n^{\circ} 8$, septembre

Granié A.M., Guetat H., (2006). "Empreintes et inventivités des femmes dans le monde rural”, PUM Toulouse, col. Ruralités Nord/Sud. 
Guillaume Le Blanc, (2007). Vies ordinaires, vies précaires, Seuil.

Hellio E., "Importer des femmes pour exporter des fraises" (Huelva), Études rurales 2008/2, I82, p. I85-200.

JACQUET, I., I995, "Développement au masculin, féminin - le genre, outils d'un nouveau concept", L'Harmattan, Paris.

Kheir Z., (2004). Forgotten women; Study on the implications of the lack of legal protection for women workers in agriculture, Foundation Center for Egyptian Women's Issue, Cairo. (Arabic reference).

Kishk H., (1996). Les ouvriers agricoles en Egypte (1953-1995) : "La situation socio-économique du travail agricole salariē", Elmahrosa livre, Le Caire (référence en arabe)

Laroussi A., (2002). "La femme rurale dans l'exploitation familiale dans le Nord-Ouest de la Tunisie-pour une sociologie de rupture". L'Harmattan.

Loutfi M.F., (1980). Rural Women: Unequal Partners in Development. International Labor Organization. Genève.

Moser, Caroline O. N. (1989). "Gender planning in the third world: Meeting practical and strategic gender needs". World Development I7, (II): 1799-825.

Sayad A., I999, "La double absence. Des illusions de l'émigré aux souffrances de l'immigré". Paris, Le Seuil.

Shalaby M. T., (1991). "The Role of Women in Egyptian Rural Development", Habitat International, Vol. 55, $^{\circ}{ }_{4}$, p. 85-104.

Simon- Kumar R., (2004). Negotiating Emancipation. The Public Sphere and Gender

Toronjo D., Les "contrats en origine" dans la production intensive des fraises à Huelva, Études rurales 2008/2, I82, p. I69-184.

World Bank, (2009). Gender in agriculture sourcebook, Washington, DC

Wresinski J. Grande pauvreté et précarité économique et sociale. JO du 28 février 1987 I987; http: //www.atd-quartmonde.org/intern/fondam/ WRES_JO87.pdf. 

